\title{
Abnormal temperature dependent elastic properties of fused silica irradiated by ultrafast lasers
}

\author{
Pieter Vlugter (1)* and Yves Bellouard (1) \\ Galatea Lab, STI/IEM, Ecole Polytechnique Fédérale de Lausanne (EPFL), 2002 Neuchâtel, Switzerland
}

(Received 27 January 2022; accepted 16 February 2022; published 4 March 2022)

\begin{abstract}
Materials with thermal-invariant elastic properties are of interest for resonant device frequency and dimensional stability of precision devices. Here, we demonstrate that the temperature coefficient of elasticity (TCE) of amorphous silica can be locally reduced using femtosecond laser irradiation. Notably, a self-organized laser-induced modification shows a decrease of the TCE beyond $50 \%$. The origin of this dramatic decrease is attributed to the presence of submicron permanent densified zones within the fine multilayer structure of the laser-modified zone.
\end{abstract}

DOI: 10.1103/PhysRevMaterials.6.033602

Amorphous silica $\left(a-\mathrm{SiO}_{2}\right)$ has unique behavior among materials in general. Unlike many other glasses and the vast majority of materials in general, it exhibits a positive temperature coefficient of elasticity (TCE), i.e., its Young's modulus increases with increasing temperature, as reported by Sosman in 1927 [1]. The cause of this unusual behavior has since been disputed. One recurring idea, proposed by Babcock in 1954 [2], is that $a-\mathrm{SiO}_{2}$ contains two types of structure, coexisting within the glass, each of which behaves normally locally, where their relative amounts change continuously with temperature and pressure. According to this model, the material undergoes a reversible displacive transformation from an elastic weak state $(\alpha$-form) to a stiffer one ( $\beta$-form), similar to cristobalite, one of the crystalline polymorphs of $\mathrm{SiO}_{2}$. While for the crystalline phases the transformation, $\alpha \rightleftharpoons \beta$, occurs in a relatively small temperature window, it would transform over a broad temperature range in the case of $a-\mathrm{SiO}_{2}$. It is widely acknowledged that the structure of $a-\mathrm{SiO}_{2}$ is not fully random and possesses short-range order $(<0.5 \mathrm{~nm})$, while the medium-range structure $(0.5-1.5 \mathrm{~nm})$ consists of a network of rings of various sizes formed by $\mathrm{SiO}_{2}$ tetrahedra [3-5], where the six-membered ring structure is the most abundant member. According to molecular dynamics simulations performed by Huang et al., these six-membered ring structures are specifically responsible for the stiffening effect caused by conformation changes of the ring structures [6]; see Fig. 1. The fourfold-coordinated tetrahedra $\mathrm{SiO}_{2}$ act as a rigid-body unit [7]. During the short-range thermal expansion, the distance between $\mathrm{Si}$ and $\mathrm{O}$ of the fourfold-coordinated tetrahedra $\mathrm{SiO}_{2}$ molecule [8] causes the six-membered ring structures

\footnotetext{
*pieter.vlugter@epfl.ch

Published by the American Physical Society under the terms of the Creative Commons Attribution 4.0 International license. Further distribution of this work must maintain attribution to the author(s) and the published article's title, journal citation, and DOI.
}

to transform from an asymmetric configuration ( $\alpha$-form) to a more symmetric, and stiffer, configuration ( $\beta$-form).

$a-\mathrm{SiO}_{2}$ 's TCE is altered while undergoing irreversible transformations toward different metastable amorphous states, a transformation mechanism coined "amorphous polyamorphism" by Grimsditch [10]. Two amorphous state families are primarily identified, namely the low-density amorphous (LDA) and high-density amorphous (HDA) states, respectively. Interestingly, like other abnormal properties (e.g., the boson peak [11], softening upon compression [12], etc.), permanently densified $a-\mathrm{SiO}_{2}$ reduces the magnitude of the TCE. Furthermore, unlike amorphous ice, $a-\mathrm{SiO}_{2}$ possesses a broad range of states, within the categories of LDA and HDA states, that can be reached through various thermodynamic paths [13] or using various intense irradiation methods that stimulate bond-breaking processes and localized reordering $[14,15]$. As there is no unique path toward a given state, different densification methods, such as fast neutron exposure [16], thermal quenching [17], and cold- and hot-compression [18], will inherently lead to a distinct densification state, an amorphous structure, and a TCE reduction level.

Here, we focus our attention on the local changes of $a-\mathrm{SiO}_{2}$ 's TCE resulting from femtosecond laser pulse bulkexposure. Thanks to their high peak-power that triggers nonlinear absorption processes, and despite having a wavelength for which the material is transparent, these lasers are capable of modifying locally transparent materials only in the focal volume $[19,20]$. In $a-\mathrm{SiO}_{2}$, the modified volume is associated with microscale material property changes, e.g., the refractive index [21], chemical susceptibility [22], thermal conductivity [23], the thermal expansion coefficient [24], and Young's modulus $[25,26]$. These laser-induced physical property changes are particularly useful in the context of monolithic microdevices, and they led to the creation of monolithic devices. For example, complex integrated photonics circuits [27], tuned resonators [26,28], optomechanical devices [29], and optofluidics [30-32], made from a single piece of material and functionalized through laser exposure sequences, were reported. Specifically, $a-\mathrm{SiO}_{2}$ is attractive for its remarkable material properties, such as its low thermal 


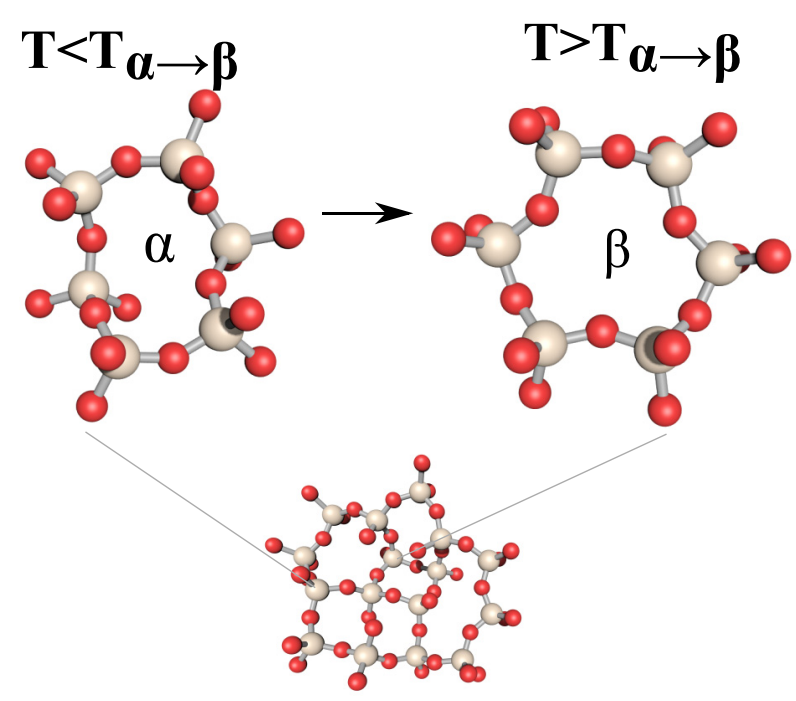

FIG. 1. An impression of the six-membered ring's conformation change from an $\alpha$ form to a $\beta$ form due to a temperature increase, adapted from [6,9]. The red and white atoms represent silicon and oxygen, respectively.

expansion, its low optical losses over a broad spectral range, as well as its low mechanical losses and high thermal stability. Unfortunately, its TCE remains non-negligible, $\sim 220 \mathrm{ppm}$ [33,34], especially in contrast with nickel-based alloys commonly referred to as Elinvar or Nivarox, which have a TCE around and below ppm levels [35]. To further enhance $a-\mathrm{SiO}_{2}$ applicability, we explore how femtosecond laser-irradiated zones can also be used to lower the intrinsic TCE value of the material. Specifically, we investigate two types of laser- induced modifications: one, commonly referred to as "type I," consists of a homogeneous modification throughout the exposed zone, and a second one, usually called "type II," consists of self-organized nanoplanes parallel to one another.

\section{EXPERIMENTAL METHODS}

TCE properties are retrieved using a method based on measuring the mechanical resonance of laser modified cantilevers as a function of temperature. We take advantage of a process based on femtosecond laser exposure and chemical etching to fabricate microcantilevers. The volume inside those cantilevers is exposed to an ultrafast laser as depicted in Fig. 2(a). The fabrication of these structures, the dimensions, and the exposure strategy are further detailed in [26].

The resonance frequency dependency on temperature, also referred to as the temperature coefficient of frequency (TCF), expressed as a function of material properties [36], is

$$
\begin{aligned}
\mathrm{TCF}=\frac{1}{f_{0}} \frac{\partial f}{\partial T} & =(\mathrm{CTE}+\mathrm{TCE}) / 2 \\
& =\left(\mathrm{CTE}+\frac{1}{E_{0}} \frac{\partial E}{\partial T}\right) / 2,
\end{aligned}
$$

where $f$ is the resonance frequency and $E$ is the Young's modulus - the subscript 0 refers to measured property at room temperature. Additionally, the TCF depends on the thermal expansion coefficient (CTE). Nevertheless, the low CTE of $a-\mathrm{SiO}_{2}\left(\sim 0.5 \mathrm{ppm} /{ }^{\circ} \mathrm{C}\right)$ makes this dependence negligible compared to the TCE $\left(\sim 220 \mathrm{ppm} /{ }^{\circ} \mathrm{C}\right)$, which is three orders of magnitude higher [33,34]. As we will see later, the laserinduced CTE changes, reported in [24], are likewise negligible compared to the laser-induced TCE changes.
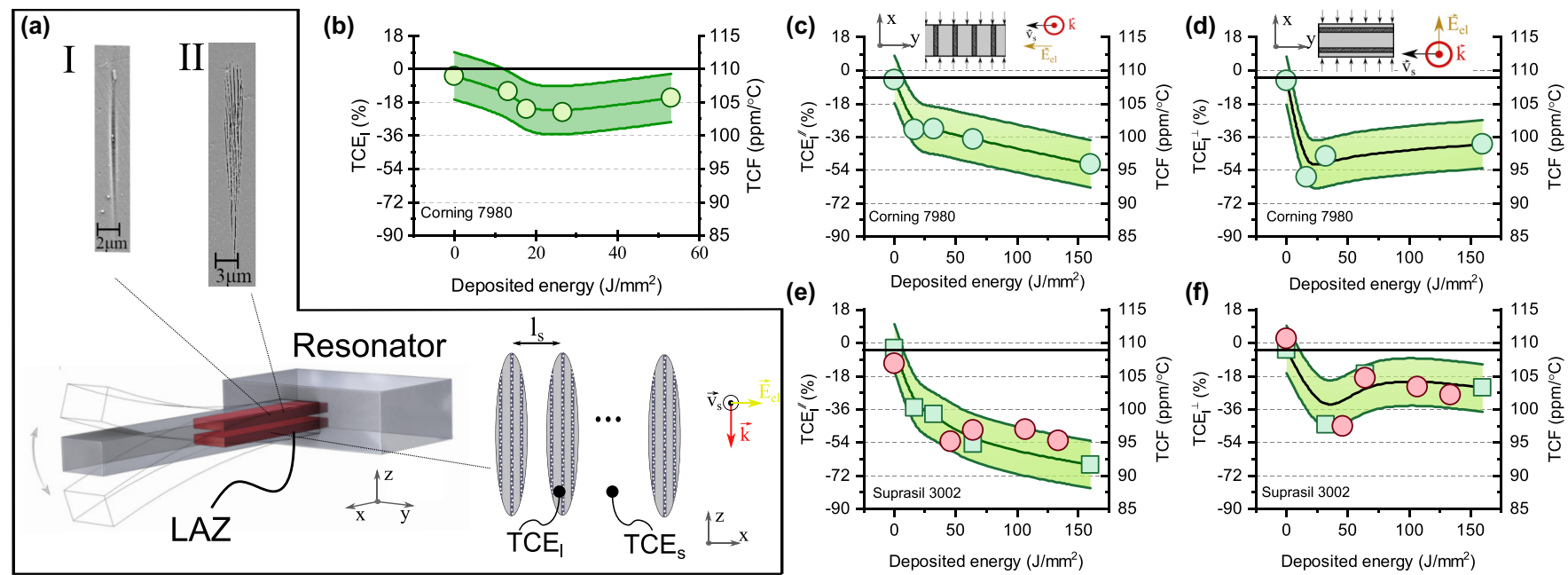

FIG. 2. (a) A schematic of the mechanical resonator with the laser affected zones (LAZs) shown in red. The insets show two scanning electron microscope (SEM) images, a typical cross-section of type I and II modifications. A schematic further depicts a set of laser modifications spaced by $l_{s}$ and where $\mathrm{TCE}_{l}$ and $\mathrm{TCE}_{s}$ indicate the TCE of the laser affected zone and of the surrounding pristine material, respectively. $\vec{v}_{s}$ is the laser writing direction, $\vec{k}$ is the optical propagation axis, and $\vec{E}_{\mathrm{el}}$ is the direction of the electric field defining the polarization of the laser. The measured TCF and related $\mathrm{TCE}_{l}$ are shown in (b)-(f). Part (b) refers to the case of a type I modification. Parts (c) and (e) show the result in the case of a type II modification for nanogratings oriented along the cantilever long-axis, while (d) and (f) show the result for a type II modification, but here with the nanogratings oriented in the transverse direction to the cantilever long-axis. The superscripts ${ }^{\perp}$ and " refers to the nanogratings orientations with respect to the cantilever long-axis. The different markers [(e) and (f)] refer to different substrates used for fabricating the resonators. 
The resonator volume is not entirely and not homogeneously exposed to the laser. Instead, the modified zones consist of a set of lines, spaced by a distance $l_{s}$ [Fig. 2(a)], where the top layer of the laser-affected volume is placed $20 \mu \mathrm{m}$ below the surface, and the bottom layer is at a depth of $300 \mu \mathrm{m}$ below the surface. The TCE extracted from the measured TCF expressed in Eq. (1) is the sum of pristine $\left(\mathrm{TCE}_{s}\right)$ and laser-affected zones $\left(\mathrm{TCE}_{l}\right)$. The change of TCE in the modified volume $\mathrm{TCE}_{l}$ is estimated based on a mathematical model, detailed in Appendix A.

The temperature is controlled and cycled between 10 and $40{ }^{\circ} \mathrm{C}$ using a dedicated environmental chamber described in [24]. The cantilever is mounted on a piezoelectric-actuator that excites it on its resonant frequency. The resonator's response is measured using a triangulation sensor (Keyence LKH022), and a phase-lock-loop scheme is applied to track the resonance frequency changes during temperature cycles. Type I modifications are produced using a femtosecond laser system emitting 50 -fs pulses at $850 \mathrm{~nm}$ and a repetition rate of $120 \mathrm{kHz}$ (Satsuma seeding an OPA from Amplitude), while type II modifications are obtained with an Yb-doped fiberamplified system emitting 380-fs pulses at $1030 \mathrm{~nm}$ and a repetition rate of $750 \mathrm{kHz}$ (Yuzu from Amplitude). In the sequel, the pulse energies used to obtain the modified zone are kept constant (250 $\mathrm{nJ}$ for type I and $240 \mathrm{~nJ}$ for type II), while the exposure dose is varied by changing the scanning speed. As for the substrates, we consider two types of $a-\mathrm{SiO}_{2}$ : one with a high $\mathrm{OH}$ content (Corning, ref. 7980) and one with a low $\mathrm{OH}$ content (Heraeus, Suprasil 3002).

\section{RESULTS}

Figure 2(b) shows the TCF and the corresponding $\mathrm{TCE}_{l}$ variation as a function of the deposited energy for type I modifications. The deposited energy is calculated as explained in [37]. With these experimental conditions, we observe a TCF decrease of $7 \mathrm{ppm} /{ }^{\circ} \mathrm{C}$ and we estimate a TCE decrease of about 20\%. Figures 2(c) and 2(d) consider the TCF and estimated TCE versus the deposited energy, which are depicted for a type II modifications for two opposite nanograting orientations. The change of TCE shows a distinct behavior in both cases. A saturating behavior for the case displayed in Figs. 2(d) and 2(f), corresponding to a transverse nanograting orientation with respect to the cantilever long-axis, is found, while a linear decay for the case in Figs. 2(c) and 2(e) is observed and corresponds to nanogratings aligned along the cantilever long-axis. This observation is useful to understand better what the origin of the reduction is, and it will be discussed more in depth in the following section. For type II modifications, the TCE reductions are of larger magnitude compared to type I modifications. For nanograting aligned along the cantilever long-axis [Figs. 2(c) and 2(e)] the reduction is eventually reaching values $\triangle \mathrm{TCE} \sim 60 \%$ for a deposited energy of $160 \mathrm{~J} / \mathrm{mm}^{2}$. The LAZ's CTE changes are negligible compared with the TCE changes, based on data reported in [24], which confirms our earlier stated hypothesis.

In [38] it was demonstrated that the focusing depth has an effect on the volume variation of the laser affected zone. This effect is related to the dispersion of the focus due to spherical aberrations. Therefore, the TCE estimate is an average, where

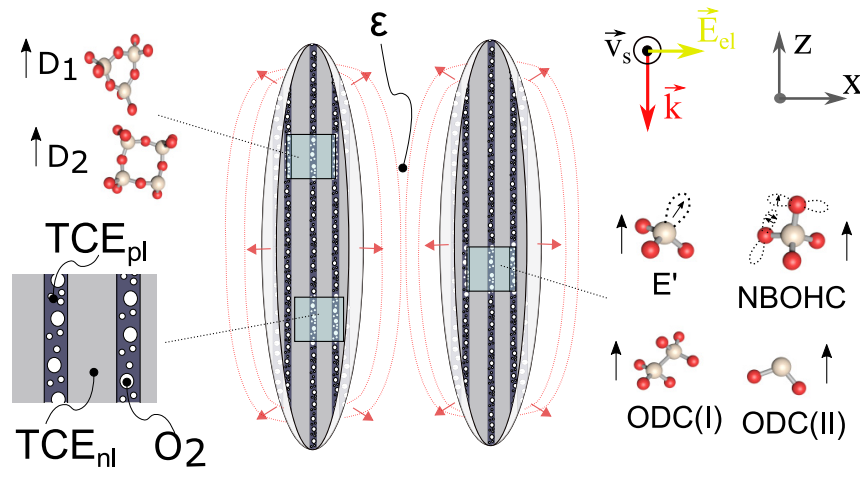

FIG. 3. Cross-section of two adjacent laser-written tracks with different regions, characteristics of a type II modification (so called "nanograting" structure). $\mathrm{TCE}_{n l}$ and $\mathrm{TCE}_{p l}$ are the temperature coefficient of elasticity of the nonporous and porous layers, respectively. The red contour map illustrates the strain induced by the laserinduced volume expansion [40]. Further, the increase of point defect density is illustrated, as is the increase of the three- and fourmembered rings.

presumably the change at the top layer is higher, while a smaller change is expected for the lower located layers.

\section{HYPOTHESIS TOWARD THE ORIGIN OF THE TCE REDUCTION}

Considering the nanogratings morphology [39] and the laser patterns considered here, several features could be responsible for the observed TCE reduction. Namely, the porous layers $\left(\mathrm{TCE}_{p l}\right)$ and/or the nonporous layers $\left(\mathrm{TCE}_{n l}\right)$, permanent densifications, elastically deformed material surrounding the LAZ $(\epsilon)$, and laser-induced point defects are possible candidates to explain the observed behavior. Figure 3(a) illustrates the characteristic laser-induced material changes that are the possible origins for the TCE reduction.

\section{A. Elastic deformation}

The porous character is responsible for a net volume expansion of the modified volume [41]. Consequently, the surrounding unexposed material is elastically densified. From compression studies in the elastic regime, it is known that the athermal Young's modulus softens [12,42]. This could indicate that the symmetric ring structures undergo a displacive transformation towards a weaker asymmetric form, due to an external pressure [6]. Following this hypothesis, one can expect that this permanent external pressure prevents this weaker asymmetric form from transforming back into its stiffer symmetric configuration. As a consequence, the TCE would reduce as the stiffening mechanism (described in the Introduction) is obstructed due to the presence of post-laser-exposure residual permanent stress. To support this hypothesis, studies on other abnormal material properties of $\mathrm{SiO}_{2}$ (i.e., boson peak [43-45]) show that elastic densification does reduce the magnitude of these abnormal behaviors. Unfortunately, to the best of our knowledge, no experimental result has been reported related to the correlation between elastic deformation and TCE. 


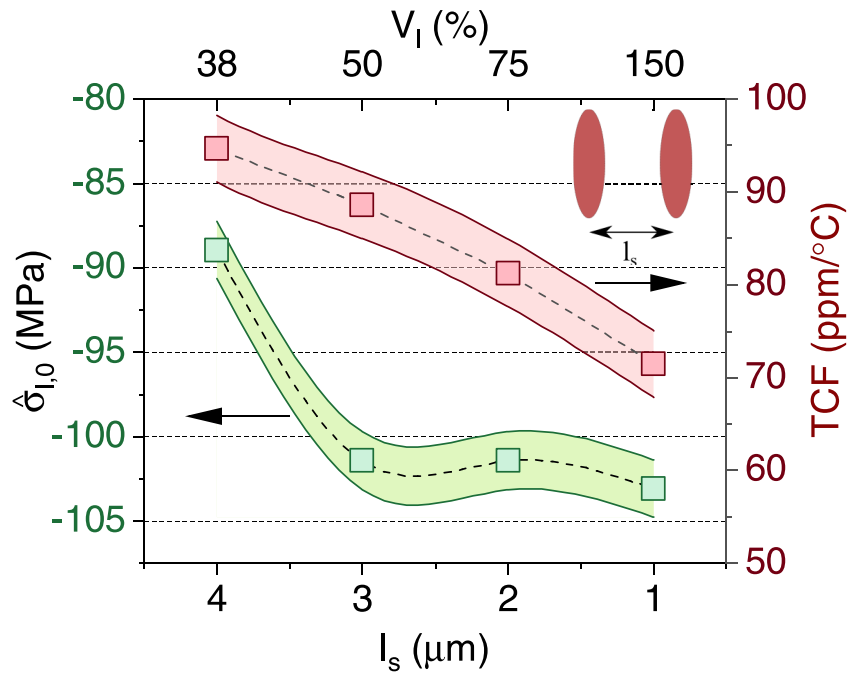

FIG. 4. Stress $\left(\hat{\sigma}_{l, 0}\right)$ and TCF vs laser-written line-spacing $\left(l_{s}\right)$ and LAZ volume fraction $\left(V_{l}\right)$. The latter is defined as the ratio between the LAZ's width and linespacing. The exposure conditions are as follows: pulse energy $300 \mathrm{~nJ}$, repetition rate $750 \mathrm{kHz}$, scanning velocity $5 \mathrm{~mm} / \mathrm{s}$, pulse width $300 \mathrm{fs}$, and laser-wavelength $1030 \mathrm{~nm}$.

In the sequel, we make a comparative study between an experiment for probing the stress present in LAZs and the one to measure the TCE as described earlier. To probe the stress, a bimorph deflection experiment is used, like in [41], where the tip displacement of a laser-exposed cantilever is related to the stress using a modified Stoney's equation as detailed in Appendix B.

Unlike the previously discussed experiments, the exposure conditions (i.e., pulse energy and exposure dose) are kept constant, but the line spacing between individual laser-written lines is varied $\left[l_{s}\right.$ in Fig. 2(a)]. This parameter effectively controls the average stress present in laser-written patterns; see Fig. 4. On the one hand, the stress seems to saturate for line-spacing below $4 \mu \mathrm{m}$ (green curve in Fig. 4), which is in agreement with our previous observations [24]. On the other hand, the TCF does not follow the same trend, but rather seems to be linearly proportional with line-spacing. There is therefore no obvious correlation between the elastic deformation of the unexposed material in between the laser-written line and the reduction of TCE. This agrees well with the MD simulations reported by Huang et al. [6], who concluded that the $\alpha-\beta$ transitions are saturated beyond the pressure window ranging from -9 to $6 \mathrm{GPa}$. Therefore, for the pressure levels obtained in our experiments, a sufficient amount of asymmetric ring-structures can be thermally transformed to their stiffer counterpart.

\section{B. Porous and nonporous layer}

At this point of the discussion, it is excluded that the elastic deformation has an effect on the observed TCE reduction. To quantify whether porous or nonporous layers are contributing to the reduction of the TCE, we revisit the results regarding the two orthogonal nanograting orientations, depicted in Fig. 2. For the nanogratings aligned along the loading direction (Fig. 2), the TCE of the nonporous layers $\left(\mathrm{TCE}_{n l}\right)$ is dominant, since it is equivalent to a mechanical parallel configuration. Furthermore, the nonporous layer is nearly a factor 10 stiffer than the porous layer and occupies nearly $90 \%$ of the volume [26]. For the orthogonal arrangements [Figs. 2(d) and 2(f)], the porous layer contribution is this time prominent, as this arrangement of layers is equivalent to in-series connected stiffness. This reasoning is supported by estimates made in Eq. (2), which considers nanogratings aligned along the loading direction, and Eq. (3), for which they are aligned transversely. For $V_{n l} \sim 0.9, V_{p l} \sim 0.1$ ([46]), and $E_{p l} \sim E_{n l} 0.1$ ([26]), Eq. (2) reveals that the behavior of $\mathrm{TCE}_{l}^{\perp}$ in Figs. 2(c) and 2(e) is mostly governed by the nonporous layer's contribution to the TCE, while for an orthogonal nanograting orientation, Eq. (3), both nonporous and porous layers contribute nearly equally, resulting in the behavior observed in Figs. 2(d) and 2(f),

$$
\begin{aligned}
\mathrm{TCE}_{l}^{/ /} & =\frac{\mathrm{TCE}_{n l} V_{n l} E_{n l}+\mathrm{TCE}_{p l} V_{p l} E_{p l}}{E_{n l} V_{n l}+E_{p l} V_{p l}} \rightarrow \\
\mathrm{TCE}_{l}^{/ /} & \sim \mathrm{TCE}_{n l} 0.9+\mathrm{TCE}_{p l} 0.01, \\
\mathrm{TCE}_{l}^{\perp} & =\frac{\mathrm{TCE}_{n l} V_{n l} E_{p l}+\mathrm{TCE}_{p l} V_{p l} E_{n l}}{V_{n l} E_{p l}+V_{p l} E_{n l}} \rightarrow \\
\mathrm{TCE}_{l}^{\perp} & \sim \mathrm{TCE}_{n l} 0.9+\mathrm{TCE}_{p l} .
\end{aligned}
$$

\section{Annealing}

To further test the hypothesis that permanently densified zones (i.e., zones within the laser modified volume and in between nanoporous layers) are the main source for TCE reduction, we investigated the behavior of the cantilever after various thermal annealing steps. Depending on the temperature at which it is performed, thermal annealing of laser affected zones has different effects. For instance, for low annealing temperatures $\left(T_{\mathrm{ann}}<500^{\circ} \mathrm{C}\right)$, laser-induced point defects [e.g., dangling bonds, nonbridging oxygen hole centers $(\mathrm{NBOHC})$, etc.] are specifically altered $[47,48]$, while for intermediate temperatures $\left(500<T_{\text {ann }}<900^{\circ} \mathrm{C}\right)$, permanent densification is quenched $[18,49]$. Finally, the porous layers are removed for annealing temperatures beyond the so called "glass annealing temperature" [50], which for the Corning 7980 glass is found at $T_{\text {ann }} \sim 1042{ }^{\circ} \mathrm{C}$. Therefore, we logically expect changes in the TCE depending on the annealing temperature that may provide further hints about the origin of the TCE reduction.

In this set of experiments, we characterized the athermal Young's modulus and the TCE of postannealed resonators under various conditions. In practice, the resonators are placed in a nitrogen-purged furnace and the temperature is elevated to a set of characteristics levels $T_{\mathrm{ann}}$. Heating and cooling rates are set below $1{ }^{\circ} \mathrm{C} / \mathrm{min}$ to avoid additional residual stress and undesired transient effects. To study the fine changes of the TCE as a function of the annealing temperature, we reuse the specimens with close-spaced exposure patterns discussed in Fig. 4. For investigating the changes in athermal Young's moduli, we reuse the samples discussed in Fig. 2(d). The athermal modulus is known to decay as a function of the deposit in an inversely proportional manner [26]. This reduction of the elastic properties is attributed to the porous planes [26]. 

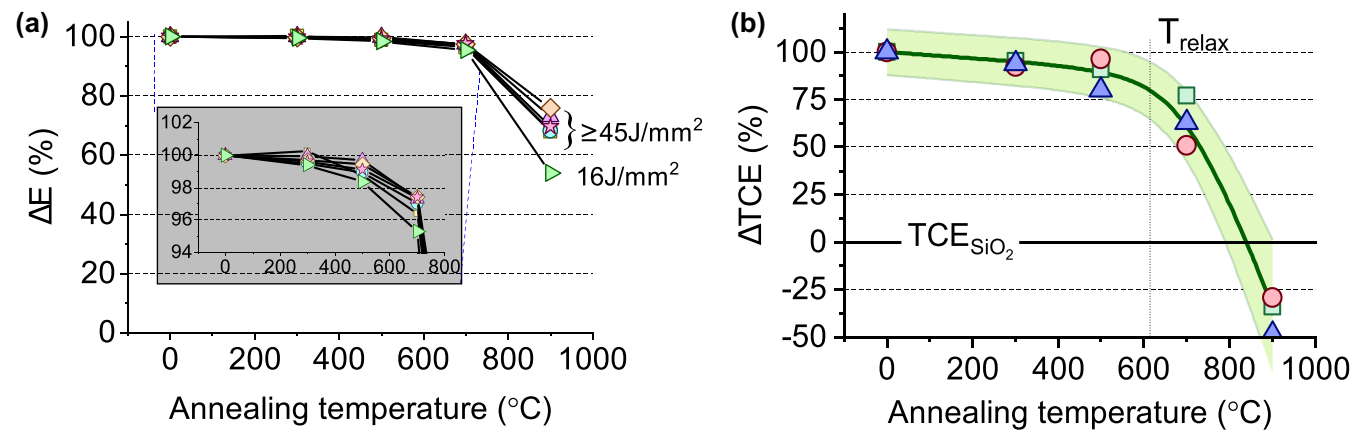

FIG. 5. (a) The change in athermal Young's modulus $(\Delta E)$ vs the annealing temperature. (b) The change in LAZ's temperature-elastic coefficient $(\triangle \mathrm{TCE})$ vs annealing temperatures.

Figures 5(a) and 5(b) depict the athermal Young's modulus (E) and TCE normalized relative changes, respectively. The references for normalization are the nonannealed laser-exposed material, meaning that $100 \%$ corresponds to the nonannealed case and $0 \%$ to the unexposed one.

Overall, the laser-induced TCE and Young's modulus changes remain almost unaffected $(<2 \%)$ for increasing annealing $T_{\text {ann }}$ up to $600{ }^{\circ} \mathrm{C}$. This indicates that point defects like $\mathrm{E}^{\prime}$ centers, NBOHC, ODC(I), and ODC(II) do not have a noticeable effect on the reduction of the TCE as their concentration gradually depletes at these annealing temperatures and durations [48,51,52]. Likewise, interstitial oxygen, found in the Raman spectrum [39,53], disappears at these annealing temperatures [54]. In [48], it is suggested that interstitial oxygen and $\mathrm{E}^{\prime}$ centers interact and form peroxy radicals (for $T_{\text {ann }}<400^{\circ} \mathrm{C}$ ). Although these chemical rearrangements very likely have an effect on the thermal elastic behavior, its significance is too small to measure due to a relatively low concentration.

The annealing experiments to $\Delta E$ [Fig. 5(a)] indicate that porous planes survive at least up to a temperature of $T_{\mathrm{ann}}<$ $900^{\circ} \mathrm{C}$. This high thermal stability of the porous planes has also been observed elsewhere using a different methodology $[55,56]$ and is further extensively studied and modeled in $[50,57]$. Contrary to the Young's modulus, the TCE recovers at $T_{\text {ann }}=900^{\circ} \mathrm{C}$. As the porous layers are not recovered at these annealing temperatures, it suggests that the nonporous layers are the main origin of TCE reduction.

From $T_{\text {ann }}=700^{\circ} \mathrm{C}$, the amplitude of $\triangle \mathrm{TCE}$ reduces significantly. At $T_{\mathrm{ann}}=900^{\circ} \mathrm{C}, \Delta \mathrm{TCE}$ is approximately $-25 \%$. This could mean that the recovered nonporous layers do not have a similar molecular arrangement to that of the pristine material, resulting in a negative $\triangle$ TCE. Another explanation is that the porous layers possess a positive $\triangle \mathrm{TCE}$, since the porous layers still exist for these annealing conditions. Effectively this would mean that the TCE reduction of the nonporous layers is higher than what we effectively measured, hence $\mathrm{TCE}_{n l}>\mathrm{TCE}_{l}$.

Raman spectroscopy studies performed by Wang et al., Witcher $\mathrm{et}$ al., and Zhang et al. monitored the $D_{1}$ and $D_{2}$ peaks as a function of the annealing temperature; these peaks are usually attributed to three- and four-membered rings structures [52,55,57]. Typically, they observed that $T_{\text {relax }}$ of the $D_{1}$ and $D_{2}$ peaks is in the range from 600 to $700{ }^{\circ} \mathrm{C}$. The annealing behavior of these densification signatures in the
Raman spectrum correlates well with the annealing behavior of the TCE found in this work. In a previous work, based on a dedicated study related to the athermal Young's modulus, we suggested that the nonporous layers in the nanograting modification are densified [26]. This fact was also reported in earlier work by Bricchi et al., but that time it was based on birefringence measurements [46].

Let us now consider the annealing behavior for specimens densified with other non-laser-based methods, namely cold[58] and hot-mechanical compression [18,49], fast neutron radiation [59], or shockwave-induced densification [60]. Hotcompressed and shockwave densification reveal similar trends for densification versus $T_{\mathrm{ann}}$, a plateau for the lower temperatures, and a decrease at higher temperatures. However, for the cold-compressed methods (where $\mathrm{SiO}_{2}$ is compressed beyond its "plastic" limit of $9 \mathrm{GPa}$ at room temperature [58]) and for a fast-neutron radiation scenario, a monotonic decrease is revealed [59]. Shockwave-induced densification reveals a relaxation temperature at $T_{\text {relax }} \sim 600^{\circ} \mathrm{C}$ [60], while for hot-compressed specimens it depends on the magnitude of the applied compression pressure [18] and temperature [49]. Cornet et al. studied the relaxation of hot-densified $\mathrm{SiO}_{2}$ using small- and wide-X-ray diffraction methods [49]. They revealed that for higher compression temperatures, the densified structure is more thermally stable (i.e., a higher $T_{\text {relax }}$ ). In their case, $T_{\text {relax }}$ ranged between 400 and $850{ }^{\circ} \mathrm{C}$ for compression temperatures ranging between 400 and $1020^{\circ} \mathrm{C}$.

The annealing behavior in this work correlates well with the annealing behavior of the densification signatures found in the Raman spectrum, as well as with other non-laser-based densification methods. This can be seen in Fig. 6, which provides a visual summary. Summarizing these observations, the evidence is strong that the TCE reduction measured in this work is due to permanent densification where parts of the material are transformed to HDA. The latter is also mentioned in [57].

To summarize the preceding subsections, we have provided evidence that the nonporous layer is the main origin for the reduction of the TCE and not the porous layer. In this scenario, the elastically deformed material due to volume changes does not play a role in the reduction of the TCE, nor do the point defects. We gave further evidence that the TCE reduction correlates well with permanent densification signatures, and therefore we suggest that the nanograting modification includes volumes that are in a high-density amorphous state. 


\begin{tabular}{l} 
Trelax $\left[{ }^{\circ} \mathrm{C}\right]$ \\
$\begin{array}{c}\text { Laser induced type II } 0 \\
\text { modification }\end{array}$ \\
\hline Point defects \& $O_{2}[48,52,54]$ \\
\hline Raman: $D_{1} \& D_{2}[52,55,57]$ \\
\hline Porous layers $[50,55]$ \\
\hline Birefrigent $[46,56]$ \\
\hline TCE [This work] \\
\hline $\begin{array}{l}\text { Non fThis work] } \\
\text { densification }\end{array}$ \\
\hline Cold-compressed $[58]$ \\
\hline Fast-neutron radiation $[59]$ \\
\hline Hot-compressed $[18,49]$ \\
\hline Shockwave $[60]$
\end{tabular}

FIG. 6. An overview of the relaxation temperatures during annealing for laser-induced material changes and for non-laser-induced densification in $a-\mathrm{SiO}_{2}$. Great care should be taken when extracting precise values from this graph, as the exact values depend on the exact material composition (e.g., $\mathrm{OH}$ or $\mathrm{Cl}$ content) [56] and annealing conditions (e.g., duration and heat-up/cool-down rate). Nevertheless, it provides a useful global overview.

Additionally, we highlight that possible densification around nanopores in the porous layer does not contribute to a measurable reduction of TCE, as observed in this work.

\section{DISCUSSION ON THE EFFECT OF THE OH CONTENT}

In these experiments (as outlined in Fig. 2), we used a wet and dry type of silica, Corning $7980(\mathrm{OH}=1000 \mathrm{ppm}$, which has a ppm-level of impurities) and Suprasil $3002(\mathrm{OH}<$ $1 \mathrm{ppm}$, which has a ppb-level of impurities), respectively. We observed that the maximum TCE reduction is slightly higher for the dry substrate (Suprasil 3002). Other studies related to nanograting formation in dry- and wet-silica reported no difference in optical retardance measurements [56], as well as in electron density of the laser-exposed plasma [61]. According to Bricci et al. [62], the retardance induced by nanograting modification is mostly governed by the large refractive index change in the porous planes. On the contrary, here the TCE reduction is attributed to the densification of the nonporous planes. Furthermore, a dependence between $\mathrm{OH}$ content and point defects (as reported in [63]) can also be ruled out, as the TCE reduction does not show evidence of a correlation with the generation of point defects.

It is known that the $\mathrm{OH}$ content reduces the viscosity of glasses [64]. Effectively, the annealing glass temperature is lower for wet-silica compared to dry-silica, and therefore it would have a lower thermal-stability compared with its dry counterpart. A possible hypothesis for this noticeable difference in TCE behavior for laser-exposed dry- and wet-silica is that the densification (TCE reduction) is quenched due to the thermal accumulation during the exposure. Morphological modifications due to thermal accumulation are clearly visible for repetition rates typically above $1 \mathrm{MHz},[65,66]$, by the appearance of the LAZ dimensions surpassing the focal volume [67], removal of the nanogratings [68], and bubble formation [66]. While in our case the repetition rate is below the $1 \mathrm{MHz}$ level, evidence of thermal accumulation based on retardance measurements (but without noticeable morphological changes) reported in [69] may suggest that a thermally

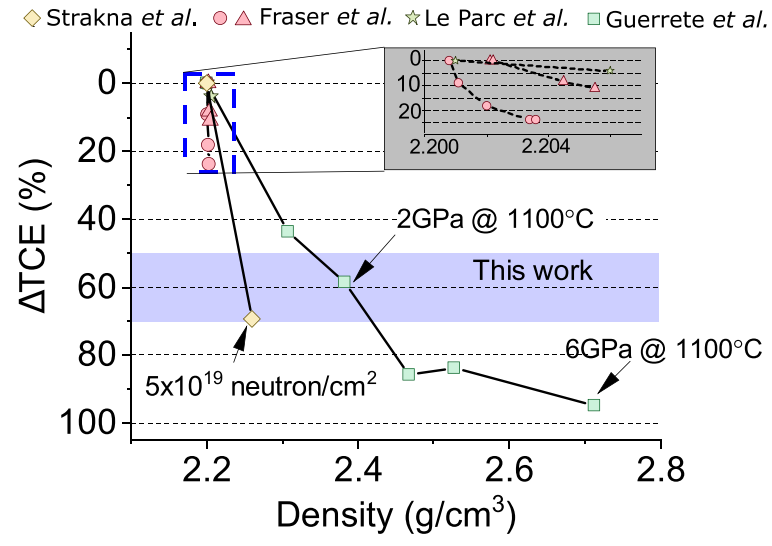

FIG. 7. A summary of $\triangle$ TCE obtained by a different densification process as reported in the literature $[16-18,75]$. The typical value obtained in this study is also shown.

cumulative effect may still be at play. Note that the cumulative exposure effect further depends on pulse energy, confocal parameters, and scanning speed [70]. While the hypothesis of a thermal effect should be considered to explain the discrepancies between dry- and wet-silica, we cannot be fully sure at this stage due to a lack of sufficient evidence. A systematic study of the TCE reduction for varying and lower repetition rates, e.g., $<0.2 \mathrm{MHz}$, would be required to confirm or refute this hypothesis. Incidentally, the use of lower repetition rates may lead to even bigger TCE reductions as the annealing effect at high repetition rates is detrimental with respect to laser-induced TCE reductions.

\section{DISCUSSION ON THE NATURE OF THE TCE REDUCTION}

Various works have demonstrated that densifying $\mathrm{SiO}_{2}$ reduces the TCE. Le Parc obtained a TCE reduction of thermally quenching $a-\mathrm{SiO}_{2}$ at atmospheric pressure [17]. Based on Brillouin scattering measurement, the author estimated a TCE reduction of $5 \%$ maximum. Strakna et al. studied the reduction of TCE for fast-neutron irradiated $a-\mathrm{SiO}_{2}$ samples in the temperature range from 100 to $300 \mathrm{~K}$ [16]. They showed that the TCE is reduced by approximately $70 \%$. In recent work, Guerrete et al. investigated the TCE for hot-compressed $a-\mathrm{SiO}_{2}[18]$ and found a TCE reduction of more than $80 \%$ for compression pressures of $6 \mathrm{GPa}$ at a temperature of $1100{ }^{\circ} \mathrm{C}$. Figure 7 summarizes the reduction of TCE obtained with various densification processes, compared with the result of this study (in the particular case of type II modification, a deposited energy of $160 \mathrm{~J} / \mathrm{mm}^{2}$, and a polarization perpendicular to the laser-scanning direction).

Based on this overview, the evidence is strong that compacting $a-\mathrm{SiO}_{2}$ results in a reduction of the TCE. In a densified material, the compaction hinders conformation changes of the six-membered rings structures, due to the lack of space $[18,71]$. Furthermore, the ring size distribution seems to have an effect on the magnitude of the TCE reduction. For instance, densification induced by ionizing radiation causes three- and four-membered rings to be generated at the expense of a decay of the number of six-membered ring structures [72,73]. As the 
three- and four-membered rings are stiffer structures, they are less prone to conformation changes [71], and consequently no stiffening effect upon a temperature increase is expected for a lower number of ring members, as this would be the case for six-membered rings in fused silica. Works on intermediate glass [71,74] suggest a correlation between the magnitude of the TCE and the amount of six-membered rings [71,74].

The TCE reduction obtained in this study seems remarkably high when comparing it with results obtained for the homogeneous modification (type I), Fig. 2(a). This indirectly suggests that the densification of the nonporous planes in the nanograting modification is higher than homogeneous modification. This is also indirectly suggested in other works, based on the study of the athermal elastic properties [26] and the refractive index [46] of the nonporous planes, where both the athermal elastic properties and the refractive index are significantly higher compared to that of the type I modification.

The onset for densification of the type I modification is generally attributed to fast cooling after laser exposure [57] or/and to a bond-breaking mechanism, induced by ionization radiation, creating smaller ring-members [73]. As reviewed above, both mechanisms-fast thermal quenching and ionizing radiation-produce similar TCE reductions to those obtained for the homogeneous modification. The result correlates well with similar densification mechanisms. Simultaneously, this raises the question of what other mechanism is taking part in reducing the TCE of the nanograting regime, and this with a magnitude of approximately $60 \%$. In [26], we envisioned that the nanograting modification's nonporous plane densification mechanism is different from the one found in type I exposure conditions. Accordingly, we further suspected that pressures generated at the porousplane location further densify the surrounding material, i.e., the nonporous planes, at elevated temperatures as the volume change of the porous planes exerts a static pressure. Based on the new insights provided here, these hypotheses might not hold. From the annealing experiments, in this work we can state that this static pressure of the porous planes does not play a role. The TCE reduction, and therefore the densification of these zones, recovers for $T_{\text {ann }}>600^{\circ} \mathrm{C}$, while the static pressure of the porous planes is still present. This observation suggests that the onset of the densification is of a temporal nature and appears during the exposure or shortly thereafter.

A precise justification for the onset for higher densification in the nonporous planes is beyond the scope of this work and deserves a more dedicated study. We would like nevertheless to point out a possible line of thought. The nanopores found in the porous layers are a product of glass decomposition due to the locally enhanced plasma density $[39,76]$. The localized enhanced electron density [77] leads to a rapid, subnanosecond [78] increase of the lattice temperature. A shockwave is generated due to fast and local thermal expansion of the material. One the one hand, the negative pressure caused by the rarefaction wave results in a tensile force onto the material and initiates a cavitation process, leading to the formation of nanopores $[79,80]$. On the other hand, at the front of the shockwave, compressive stress exists. This compressive stress might be the cause of this high TCE reduction found in a nonporous layer.

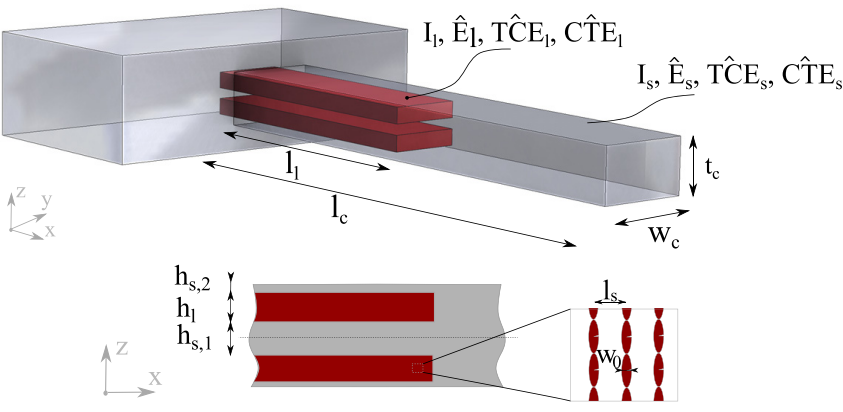

FIG. 8. A schematic drawing of the resonator with the LAZ shown in red. The dimensions, second moment of area $I$, and material properties (E., TCE., CTE.).

\section{CONCLUSION}

With our earlier work in Ref. [24], which described the behavior of the coefficient of thermal expansion, and Ref. [26], which addressed the change in Young's modulus at room temperature, this work finalizes a thermomechanical description of femtosecond irradiation effects on $a-\mathrm{SiO}_{2}$ by providing insights related to the laser-induced changes induced to the temperature coefficient of elasticity (TCE).

Here, we observed that the local decrease of the temperature coefficient of elasticity of $a-\mathrm{SiO}_{2}$ exposed to femtosecond laser irradiation can be significant, eventually reaching $60 \%$ and among the highest reported effects for the various methods reported to decrease the TCE of silica.

These findings demonstrate the opportunity of tailoring the thermomechanical behavior of dielectrics in a noncontact and localized manner. This is useful for, e.g., watchmaking, frequency references, and precision devices, which require high dimensional stability. Furthermore, the applied methodology in this work can be used for exploring the TCE reductions in other glass formers using ultrafast laser.

\section{ACKNOWLEDGMENT}

The Galatea Lab acknowledges the sponsorship of Richemont International SA.

\section{APPENDIX A: DERIVATION OF RESONANCE FREQUENCY-MATERIAL PROPERTY RELATION}

In this Appendix, we express the resonator's resonance frequency in terms of the material properties of both the laserexposed and pristine material, since it is partly exposed (see Fig. 8). The lowest resonant frequency of a clamped-free cantilever is expressed in Eq. (A1). $\rho$ and $V$ are the mass density and the volume of the cantilever. E. and I. are the Young's modulus and the second moment of inertia, respectively, with the subscripts.$l$ and ${ }_{s}$ referring to the laser affected zones (LAZ) and the nonexposed ones, respectively. The circumflex as a diacritical mark indicates that the variable is a volume average, and the subscript ${ }_{0} 0$ indicates a temperature invariant variable. $v$ is the wave number, which corresponds to a 
resonance mode,

$$
f=\frac{v^{2}}{2 \pi} \sqrt{\frac{\hat{E}_{l} I_{l}+E_{s} I_{s}}{\rho V l^{3}}}
$$

with $\rho=\rho_{0} /(1+\mathrm{CTE} \Delta T), \quad V=V_{0}(1+\mathrm{CTE} \Delta T)$, $l=l_{0}\left(1+\mathrm{C}^{\mathrm{T} E} E_{l} \Delta T\right), \quad I_{l}=I_{l, 0}\left(1+\mathrm{CTE}_{l} \Delta T\right)^{4}, \quad$ and $I_{s}=I_{s, 0}\left(1+\mathrm{CTE}_{s} \Delta T\right)^{4}$. Equation (A2) becomes

$$
f=\frac{v^{2}}{2 \pi} \sqrt{\frac{\hat{E}_{l} I_{l, 0}\left(1+\mathrm{CTE}_{l} \Delta T\right)+E_{s} I_{s, 0}\left(1+\mathrm{CTE}_{s} \Delta T\right)}{\rho_{0} V_{0} l_{0}^{3}} .}
$$

The temperature-dependent elastic constants are linearized to the first order, i.e., $E_{s}=E_{s, 0}+d E_{s} / d T \Delta T$. The thermal derivative of the frequency, evaluated at $\Delta T=0$, is

$$
\begin{aligned}
\left.\frac{\partial f}{\partial T}\right|_{\Delta T=0}= & \frac{v}{4 \pi} \frac{1}{\sqrt{\hat{E}_{l, 0} I_{l, 0}+E_{s, 0} I_{s, 0}}} \frac{1}{\sqrt{l_{0}^{3} \rho_{0} V_{0}}} \\
& \times\left[I_{l, 0}\left(\frac{d \hat{E}_{l}}{d T}+\hat{E}_{l, 0} \mathrm{CTE}_{l}\right)\right. \\
& \left.+I_{s, 0}\left(\frac{d \hat{E}_{l}}{d T}+E_{s, 0} \mathrm{CTE}_{s}\right)\right] .
\end{aligned}
$$

Simplifying further this equation, with the assumption that the terms containing the coefficient of thermal expansion (CTE.) are much smaller (by an order of magnitude $\sim 4$ ) than the ones containing elastic constant thermal derivatives $(\partial E . / \partial T)$, gives

$$
\left.\frac{\partial f}{\partial T}\right|_{\Delta T=0}=\frac{f_{0}}{2} \frac{1}{\hat{E}_{l, 0} I_{l, 0}+E_{s, 0} I_{s, 0}}\left[I_{l, 0}\left(\frac{d \hat{E}_{l}}{d T}\right)+I_{s, 0}\left(\frac{d E_{s}}{d T}\right)\right] .
$$

Rewriting the equation in terms of TCE. (i.e., $d E_{l} / d T=$ $E_{l, 0} \mathrm{TCE}_{l}$ ) yields

$$
\left.\frac{\partial f}{\partial T}\right|_{\Delta T=0}=\frac{f_{0}}{2}\left[\frac{I_{l, 0} \hat{E}_{l, 0} \mathrm{TCE}_{l}+I_{s, 0} E_{s, 0} \mathrm{TCE}_{s}}{\hat{E}_{l, 0} I_{l, 0}+E_{s, 0} I_{s, 0}}\right] .
$$

$\mathrm{TCE} E_{l}$ and $\hat{E}_{l, 0}$ are the average properties of the modified volume, which are further specified in Eqs. (A6) and (A7), in terms of the volume fractions, $V_{l}$ and $V_{s}$, respectively, and where LAZ and the pristine volume fraction are expressed as $V_{l}=w_{0} / l_{s}$ and $V_{s}=1-V_{l}$ (see Fig. 8),

$$
\begin{gathered}
\mathrm{TCE} E_{l}=\frac{\mathrm{TCE}_{s} V_{s} E_{l, 0}+\mathrm{TCE}_{l} V_{l} E_{s, 0}}{V_{s} E_{l, 0}+V_{l} E_{s, 0}}, \\
\hat{E}_{l, 0}=\frac{E_{l, 0} E_{s, 0}}{E_{l, 0} V_{s}+E_{s, 0} V_{l}} .
\end{gathered}
$$

[1] D. Uhlmann, Elasticity and Strength in Glasses: Glass: Science and Technology (Elsevier, Amsterdam, 2012).

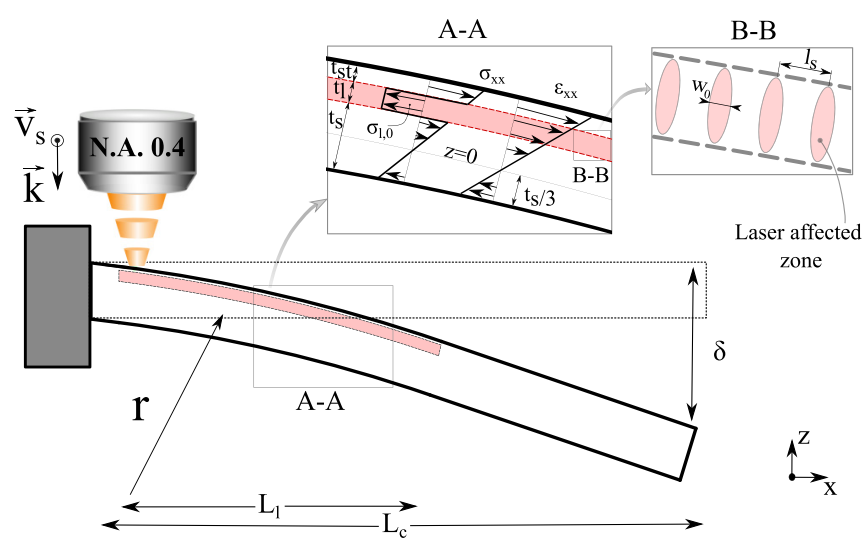

FIG. 9. A schematic of a laser-exposed cantilever. The physical dimensions are indicated along with the stress and strain profiles across the thickness. $\hat{\sigma}_{l, 0}$ is the stress in the laser-exposed layer.

\section{APPENDIX B: DERIVATION OF THE STRESS IN THE BIMORPH CONFIGURATION}

Here, we derive a mathematical expression for the stress in the modified layer $\hat{\sigma}_{l, 0}$ of the laser-written bimorph structure. In Fig. 9, a schematic is depicted of a cantilever being exposed just below the top-layer and consequently bending with a bending radius $r$ due to volume expansion of the laser affected zone. The stress and strain profile is illustrated in the inset A-A of Fig. 9, where the stress in the laser-affected layer, $\hat{\sigma}_{l, 0}$, is opposite in sign compared with the stress in the pristine bulk. For this system, the stress and strain in the pristine bulk is zero at a distance $t_{s} / 3$ from the bottom surface, defining the neutral axis [81]. We further define the neutral axis as the origin of the $z$-coordinate, i.e., $z=0$. The relationship between the bending radius and strain reads

$$
\epsilon_{x x}(z)=\frac{z}{r} \text {. }
$$

Newton's third law is used to find the analytical expression of $\hat{\sigma}_{l, 0}$ [Eq. (B2)]. The first two terms represent the force in the pristine bulk, with $E_{s}$ denoting Young's modulus of this volume, while the last term represents the force within the LAZ,

$$
0=\frac{\partial \epsilon_{x x}(z)}{\partial z} E_{s}\left(\int_{-t_{s} / 3}^{t_{s} 2 / 3} z d z+\int_{t_{s} 2 / 3+t_{l}}^{t_{s} 2 / 3+t_{l}+t_{s, t}} z d z\right)+\hat{\sigma}_{l, 0} t_{l} .
$$

Rewriting Eq. (B2) yields the following expression for $\hat{\sigma}_{l, 0}$ :

$$
\hat{\sigma}_{l, 0}=-\frac{E_{s} t_{s}^{2}}{6 t_{l} r}-\frac{E_{s}}{t_{l} r_{0}}\left[\frac{1}{2}\left(t_{s} \frac{2}{3}+t_{l}+t_{s, t}\right)^{2}-\frac{1}{2}\left(\frac{t_{s}}{3}+t_{l}\right)^{2}\right] .
$$

Note that the first term is the classical Stoney's equation [82]. 
[3] W. H. Zachariasen, J. Am. Chem. Soc. 54, 3841 (1932).

[4] S. V. King, Nature (London) 213, 1112 (1967).

[5] F. L. Galeener, Solid State Commun. 44, 1037 (1982).

[6] L. Huang and J. Kieffer, Phys. Rev. B 69, 224203 (2004).

[7] K. D. Hammonds, M. T. Dove, A. P. Giddy, V. Heine, and B. Winkler, Am. Mineral. 81, 1057 (1996).

[8] Y. Shi, D. Ma, A. P. Song, B. Wheaton, M. Bauchy, and S. R. Elliott, J. Non-Cryst. Solids 528, 119760 (2020).

[9] B. Deng, Y. Shi, and F. Yuan, Materialia 12, 100752 (2020).

[10] M. Grimsditch, Phys. Rev. Lett. 52, 2379 (1984).

[11] T. Nakayama, Rep. Prog. Phys. 65, 1195 (2002).

[12] P. W. Bridgman, Am. J. Sci. s5-10, 359 (1925).

[13] P. F. McMillan, M. Wilson, M. C. Wilding, D. Daisenberger, M. Mezouar, and G. Neville Greaves, J. Phys.: Condens. Matter 19, 415101 (2007).

[14] R. E. Schenker and W. G. Oldham, J. Appl. Phys. 82, 1065 (1997).

[15] I. Reghioua, M. Lancry, O. Cavani, S. L. Floch, D. R. Neuville, and N. Ollier, Appl. Phys. Lett. 115, 251101 (2019).

[16] R. E. Strakna, A. E. Clark, D. L. Bradley, and W. M. Slie, J. Appl. Phys. 34, 1439 (1963).

[17] R. Le. Parc, C. Levelut, J. Pelous, V. Martinez, and B. Champagnon, J. Phys.: Condens. Matter 18, 7507 (2006).

[18] M. Guerette, M. R. Ackerson, J. Thomas, E. B. Watson, and L. Huang, J. Chem. Phys. 148, 194501 (2018).

[19] B. C. Stuart, M. D. Feit, A. M. Rubenchik, B. W. Shore, and M. D. Perry, Phys. Rev. Lett. 74, 2248 (1995).

[20] E. N. Glezer, M. Milosavljevic, L. Huang, R. J. Finlay, T.-H. Her, J. P. Callan, and E. Mazur, Opt. Lett. 22, 422 (1997).

[21] K. M. Davis, K. Miura, N. Sugimoto, and K. Hirao, Opt. Lett. 21, 1729 (1996).

[22] A. Marcinkevičius, S. Juodkazis, M. Watanabe, M. Miwa, S. Matsuo, H. Misawa, and J. Nishii, Opt. Lett. 26, 277 (2001).

[23] Y. Bellouard, E. Barthel, A. A. Said, M. Dugan, and P. Bado, Opt. Express 16, 19520 (2008).

[24] P. Vlugter, E. Block, and Y. Bellouard, Phys. Rev. Mater. 3, 053802 (2019).

[25] Y. Bellouard, T. Colomb, C. Depeursinge, M. Dugan, A. A. Said, and P. Bado, Opt. Express 14, 8360 (2006).

[26] P. Vlugter and Y. Bellouard, Phys. Rev. Mater. 4, 023607 (2020).

[27] K. Minoshima, A. Kowalevicz, E. Ippen, and J. Fujimoto, Opt. Express 10, 645 (2002).

[28] T. Yang and Y. Bellouard, Phys. Rev. Appl. 7, 064002 (2017).

[29] Y. Bellouard, A. Said, and P. Bado, Opt. Express 13, 6635 (2005).

[30] F. He, Y. Liao, J. Lin, J. Song, L. Qiao, Y. Cheng, and K. Sugioka, Sensors 14, 19402 (2014).

[31] M. Haque, K. K. C. Lee, S. Ho, L. A. Fernandes, and P. R. Herman, Lab Chip 14, 3817 (2014)

[32] A. Schaap, Y. Bellouard, and T. Rohrlack, Biomed. Opt. Express 2, 658 (2011).

[33] S. Spinner, J. Am. Ceram. Soc. 39, 113 (1956).

[34] Y. Bao and G. Chen, Meas. Sci. Technol. 27, 065101 (2016).

[35] M. Kikuchi, H. Masumoto, and T. Nakayama, Trans. Jpn. Inst. Met. 28, 585 (1987).

[36] R. Melamud, S. A. Chandorkar, B. Kim, H. K. Lee, J. C. Salvia, G. Bahl, M. A. Hopcroft, and T. W. Kenny, J. Microelectromech. Syst. 18, 1409 (2009).
[37] S. Rajesh and Y. Bellouard, Opt. Express 18, 21490 (2010).

[38] C.-E. Athanasiou and Y. Bellouard, Micromachines 6, 1365 (2015).

[39] M. Lancry, B. Poumellec, J. Canning, K. Cook, J.-C. Poulin, and F. Brisset, Laser Photon. Rev. 7, 953 (2013).

[40] A. Champion, M. Beresna, P. Kazansky, and Y. Bellouard, Opt. Express 21, 24942 (2013).

[41] A. Champion and Y. Bellouard, Opt. Mater. Express 2, 789 (2012).

[42] T. Deschamps, J. Margueritat, C. Martinet, A. Mermet, and B. Champagnon, Sci. Rep. 4, 7193 (2014).

[43] T. Deschamps, C. Martinet, D. de Ligny, J. L. Bruneel, and B. Champagnon, J. Chem. Phys. 134, 234503 (2011).

[44] T. Deschamps, C. Martinet, D. R. Neuville, D. de Ligny, C. Coussa-Simon, and B. Champagnon, J. Non-Cryst. Solids 355 2422 (2009).

[45] R. J. Hemley, H. K. Mao, P. M. Bell, and B. O. Mysen, Phys. Rev. Lett. 57, 747 (1986).

[46] E. Bricchi and P. G. Kazansky, Appl. Phys. Lett. 88, 111119 (2006).

[47] L. Skuja, M. Hirano, H. Hosono, and K. Kajihara, Phys. Status Solidi C 2, 15 (2005).

[48] H.-B. Sun, S. Juodkazis, M. Watanabe, S. Matsuo, H. Misawa, and J. Nishii, J. Phys. Chem. B 104, 3450 (2000).

[49] A. Cornet, C. Martinet, V. Martinez, and D. de Ligny, J. Chem. Phys. 151, 164502 (2019).

[50] M. Cavillon, Y. Wang, B. Poumellec, F. Brisset, and M. Lancry, Appl. Phys. A 126, 876 (2020).

[51] A. Zoubir, C. Rivero, R. Grodsky, K. Richardson, M. Richardson, T. Cardinal, and M. Couzi, Phys. Rev. B 73, 224117 (2006).

[52] J. J. Witcher, W. J. Reichman, L. B. Fletcher, N. W. Troy, and D. M. Krol, OSA Opt. Mater. Express 3, 502 (2013).

[53] Y. Bellouard, A. Champion, B. McMillen, S. Mukherjee, R. R. Thomson, C. Pépin, P. Gillet, and Y. Cheng, Optica 3, 1285 (2016).

[54] F. Zimmermann, Ph.D. thesis, Friedrich-Schiller-Universitat Jena (2017).

[55] F. Zhang, Y. Yu, C. Cheng, Y. Dai, H. Zhang, and J. Qiu, Appl. Phys. B 117, 53 (2014).

[56] S.-E. Wei, Y. Wang, H. Yao, M. Cavillon, B. Poumellec, G.-D. Peng, and M. Lancry, Sens. Fiber Opt. Sens. Appl. XI 20, 762 (2020).

[57] Y. Wang, M. Cavillon, N. Ollier, B. Poumellec, and M. Lancry, Phys. Status Solidi A 218, 2100023 (2021).

[58] M. Grimsditch, Phys. Rev. B 34, 4372 (1986).

[59] W. Primak, Phys. Rev. 110, 1240 (1958).

[60] M. Okuno, B. Reynard, Y. Shimada, Y. Syono, and C. Willaime, Phys. Chem. Miner. 26, 304 (1999).

[61] M. Lancry, N. Groothoff, S. Guizard, W. Yang, B. Poumellec, P. G. Kazansky, and J. Canning, J. Non-Cryst. Solids 355, 1057 (2009).

[62] E. Bricchi, B. G. Klappauf, and P. G. Kazansky, Opt. Lett. 29, 119 (2004).

[63] J. Qian, G. Wang, D. Shen, K. Lou, Q. Fu, Z. Xia, and Q.-Z. Zhao, Opt. Mater. Express 10, 1241 (2020).

[64] R. Bruckner, J. Non-Cryst. Solids 5, 123 (1970).

[65] C. B. Schaffer, J. F. García, and E. Mazur, Appl. Phys. A 76, 351 (2003). 
[66] Y. Bellouard and M.-O. Hongler, Opt. Express 19, 6807 (2011).

[67] E. O. Kissi and Y. Bellouard, Opt. Express 26, 14024 (2018).

[68] S. Richter, M. Heinrich, S. Döring, A. Tünnermann, S. Nolte, and U. Peschel, J. Laser Appl. 24, 042008 (2012).

[69] M. Lancry, F. Zimmerman, R. Desmarchelier, J. Tian, F. Brisset, S. Nolte, and B. Poumellec, Appl. Phys. B 122, 66 (2016).

[70] H. Shen, H. Wang, and C. Tian, J. Heat Transf. 143, 022102 (2021).

[71] L. Huang, F. Yuan, M. Guerette, Q. Zhao, and S. Sundararaman, J. Mater. Res. 32, 174 (2017).

[72] S. Vukelić, P. Kongsuwan, S. Ryu, and Y. L. Yao, J. Manuf. Sci. Eng. 132, 061013 (2010).

[73] N. S. Shcheblanov and M. E. Povarnitsyn, Europhys. Lett. 114, 26004 (2016).
[74] M. K. Rabia, S. Degioanni, C. Martinet, J. Le Brusq, B. Champagnon, and D. Vouagner, J. Phys.: Condens. Matter 28, 075402 (2016).

[75] D. B. Fraser, J. Appl. Phys. 39, 5868 (1968).

[76] V. R. Bhardwaj, E. Simova, P. P. Rajeev, C. Hnatovsky, R. S. Taylor, D. M. Rayner, and P. B. Corkum, Phys. Rev. Lett. 96, 057404 (2006).

[77] A. Rudenko, J.-P. Colombier, and T. E. Itina, Phys. Rev. B 93, 075427 (2016).

[78] N. M. Bulgakova, V. P. Zhukov, S. V. Sonina, and Y. P. Meshcheryakov, J. Appl. Phys. 118, 233108 (2015).

[79] A. Rudenko, J.-P. Colombier, and T. E. Itina, Phys. Chem. Chem. Phys. 20, 5887 (2018).

[80] A. Rudenko, J.-P. Colombier, T. E. Itina, and R. Stoian, Adv. Opt. Mater. 9, 2100973 (2021).

[81] C.-H. Hsueh, Appl. Phys. 91, 9652 (2002).

[82] G. G. Stoney and C. A. Parsons, Proc. R. Soc. London, Ser. A 82, 172 (1909). 